\title{
Ars est artem celare - Die Lüge als rhetorische Kunst betrachtet
}

Ars est artem celare - Le mensonge considéré comme un art rhétorique Ars est artem celare - Lying considered as a rhetorical art

\section{Gert Ueding}

\section{(2) OpenEdition}

\section{Journals}

Édition électronique

URL : http://journals.openedition.org/ceg/1685

DOI : $10.4000 /$ ceg. 1685

ISSN : 2605-8359

Éditeur

Presses Universitaires de Provence

Édition imprimée

Date de publication : 1 décembre 2014

Pagination : 75-90

ISSN : 0751-4239

Référence électronique

Gert Ueding, "Ars est artem celare - Die Lüge als rhetorische Kunst betrachtet 》, Cahiers d'Études Germaniques [Online], 67 | 2014, Online erschienen am: 17 Dezember 2017, abgerufen am 04 November 2020. URL : http://journals.openedition.org/ceg/1685; DOI : https://doi.org/10.4000/ceg. 1685 


\title{
Ars est artem celare - Die Lüge als rhetorische Kunst betrachtet
}

\author{
Gert UEDING \\ Eberhard-Karls-Universität Tübingen
}

\begin{abstract}
Dass wir lügen - dieser Vorwurf ist Dichtern und Rednern gleicherweise gemacht worden, in der Regel auch von denselben Autoren: von Philosophen zumal aber auch von Kirchenvätern, in der Moderne von Wissenschaftlern, die sich als exakt definieren. Die Dichter konnten damit zumeist gut leben, das Erfreuen und Bewegen (delectare und movere) blieb ihnen allemal und nützen (prodesse) konnten sie auch auf dem Wege scheinhafter Vorspiegelung, insofern das Lügen und dessen Folgen ein lehrreiches Thema von Komödie und Tragödie gleichermaßen blieb. Aber für die Rhetorik ging es um's Ganze, da ihrem Selbstverständnis als die Wissenschaft, auf überzeugende Weise gut $\mathrm{zu}$ reden, mit dieser Beschuldigung pauschal widersprochen wurde. Platon war, wie bekannt, ihr erster prominenter Vertreter und die spitzfindigste (um nicht zu sagen: sophistische) Begründung gab er in den Dialogen Gorgias und Sophistes, unbeschadet der Tatsache, dass Sokrates den Alten selber als Vertreter sophistischer Redekunst galt, wie man sehr schön Xenophons Dialogen entnehmen kann. Den platonischen Sokrates hinderte das natürlich nicht, die Rhetorik generell unter Lügenverdacht zu stellen, sie als Schattenbild eines Teils der Staatskunst sogar böse zu nennen. ${ }^{1}$ Sie sei dem Bürger bloß gefällig, spreche dem Volke nach dem Munde, gebe den Schein für Wahrheit aus ${ }^{2}$ und stütze sich auf bloße Meinungen, noch dazu in trügerischer Absicht. ${ }^{3}$ Diese Vorwürfe sind jahrhundertelang durch die anti-rhetorische Literatur Europas gewandert, wurden oft wörtlich, wie z.B. von Immanuel Kant in der Kritik der Urteilskraft $t^{4}$, übernommen oder nur geringfügig variiert.
\end{abstract}

\footnotetext{
${ }^{1}$ PLAton, Sämtliche Werke in 3 Bänden, übers. von Friedrich Schleiermacher, Berlin, o.J., Bd. 1, S. 217 (463bff).

${ }^{2}$ Ibid., Bd. 4, S. 204 (233b ff).

${ }^{3}$ Ibid., Bd. 4, S. 234f (267a ff).

${ }^{4}$ Immanuel KANT, Kritik der Urteilskraft, Hamburg, Meiner, 1959, S. $183 \mathrm{ff}$.
} 
Jeden Leser des Gorgias- oder Sophistes- Dialogs muss es erstaunen, dass Platon aber seinerseits den Grundriss einer Rhetorik formuliert, die nun alle die fundamentalen Mängel nicht besitzen soll, die er vorher kritisiert hat. Es gelingt ihm durch einen Trick und durch die Depontiierung der Redekunst. Wer die Wahrheit suche sei bei der Philosophie an der einzig richtigen Adresse, da sie „die wahre Beschaffenheit eines jeden Dinges“"5 erkennen könne. Für die lehrende Vermittlung des „Gerechten, Schönen und Guten“ ${ }^{\text {“6 }}$ wäre dann die Rhetorik gut genug, die damit in didaktischer Funktion aufgeht. Adorno hat diesen Trick als eine Selbsttäuschung der Philosophie offengelegt, und zwar schon in den 60er Jahren des 20. Jahrhunderts, als die Wiederentdeckung der Rhetorik erst zaghaft begonnen hatte. „Durch die sei's offenbare, sei's latente Gebundenheit an Texte", schreibt er in seiner Negativen Dialektik,

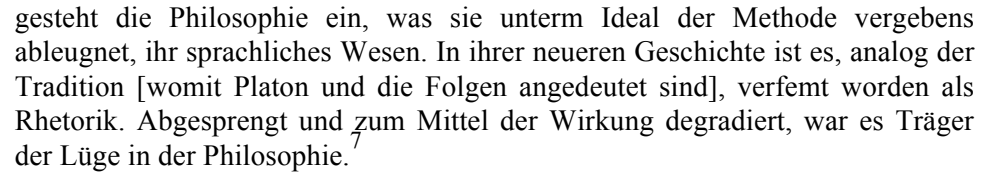

Nun, es gab Ausnahmen, und wir wollen sie gerade heute im Rahmen unseres Themas, nicht vergessen. Die früheste und was ihre historische Wirksamkeit als Gegenbewegung gegen Platon betrifft, auch die bedeutendste Ausnahme repräsentiert die Rhetorik seines Schülers Aristoteles. Der rettete die rhetorische Disziplin nun nicht, wie Adorno vorschlägt, geradewegs über die Abhängigkeit der Philosophie von Sprache, sondern über den ihr eigenen Gegenstandsbereich:

\begin{abstract}
Sie beschäftigt sich aber mit solchen Dingen, welche Gegenstand unserer Beratung sind, für die wir aber keine systematischen Wissenschaften besitzen, und vor solchen Zuhörern, die nicht in der Lage sind, vielerlei mit einem Blick zusammenzufassen und weitreichende logische Schlüsse zu ziehen. Aber wir beraten nur über solche Dinge, welche sich allem Anschein nach auf zweierlei Weise verhalten können: Denn über das, was nicht anders sein, werden oder sich verhalten kann, beratschlagt niemand, sofern er annimmt, daß es sich so verhält: das bringt ja nichts mehr ein.
\end{abstract}

Das ist der erfrischend praktische Blick, der Aristoteles so auszeichnet, weil er in keine praktizistische Verengung führt, sondern das ganze weite Problemfeld des menschlichen Handelns für das Denken, die theoretische Reflexion zugänglich macht. Auf diese Weise wird die Rhetorik zu dem Vermögen „bei jedem Gegenstand das möglicherweise Glaubenerweckende zu erkennen" und öffentlich zu vertreten. ${ }^{9}$ Ist damit aber auch jener

\footnotetext{
${ }^{5}$ Platon, Sämtliche Werke, Bd. 4, S. 57 (277b).

${ }^{6}$ Ibid., Bd. 4, S. 58 (278a).

${ }^{7}$ Theodor W. ADORNO, Negative Dialektik, Frankfurt /M., Suhrkamp, 1970, S. 65.

${ }^{8}$ ARISTOTELES, Rhetorik, München, W. Fink, 1980, S. 16 (1357a).

${ }^{9}$ Ibid., S. 12 (1355b).
} 
Lügenverdacht abgewendet, den Platon so entschieden erhob? Keineswegs, ja man könnte sogar auf die Idee kommen, dass er an Brisanz zugenommen hat. Die Formulierung („das möglicherweise Glaubenerweckende“) ist doch, sagen wir es etwas flapsig: watteweich, zumindest aber vorsichtig. Intersubjektive Gültigkeit erscheint danach für rhetorische Aussagen zumindest zweifelhaft. Aristoteles musste eine Möglichkeit finden, sie verbindlich zu machen. Das war nach der sophistischen Aufklärung schwierig geworden, die Götter konnte man dafür nicht mehr aus dem philosophischen Maschinenboden holen. „Von den Göttern vermag ich nichts festzustellen, weder, da $\beta$ es sie gibt, noch, was für eine Gestalt sie haben." ${ }^{\text {“10 }}$ Das ist Protagoras, der bedeutendste Theoretiker der Rhetorik vor Aristoteles. Und er ist auch vor der daraus folgenden Konsequenz nicht zurückgeschreckt, dass nämlich in allen Fragen des Wissens keine andere Instanz zu entscheiden hat als der Mensch selber, er ist der Maßstab jeder Erkenntnis - so der Sinn des vielbesprochenen „Homo-mensura“-Satzes. ${ }^{11}$

Die platonischen Rückzugsgefechte (Wahrheit kommt allein den Ideen zu) waren für Aristoteles nicht mehr als eben das. Er sah sie als Verlegenheit und aus dieser Verlegenheit heraus gelang ihm eine großartige Entdeckung. In einem ersten Schritt erklärte er für die Allgemeingültigkeit rhetorischer Aussagen die Adressaten (die Polisbürger) für zuständig, kehrte also das bisherige Schema um, das darin bestand, die Beweislast nach außen (an die Götter oder die Ideen) zu delegieren. Zweitens gewann er durch diese Umkehrung ein zuverlässiges Instrument der Überzeugung. Lassen wir ihn selber sprechen. Wann nämlich, so befragt er die Erfahrung erscheint ihnen, den Adressaten, eine Aussage überzeugender, wahrscheinlicher als eine andere? Das ist dann der Fall, wenn sich auf jene Sätze bezieht, „die Allen oder den Meisten oder den Weisen wahr scheinen, und auch von den Weisen entweder Allen oder den Meisten oder den Bekanntesten und Angenehmsten. “12 In dieser Form setzt Aristoteles seine bis heute virulente Entdeckung an den Anfang der Topik, der Schrift also, in der er die rhetorischen Schlussverfahren untersucht. Mit anderer Wendung werden wir ihr nochmals begegnen, in politischem Zusammenhang nämlich, wo auch die fragwürdige (des Fragens würdige) Seite dieses auf Konsens beruhenden Gültigkeitsprinzips diskutiert und einer wiederum überraschenden Lösung zugeführt wird.

Für den gegenwärtigen Zusammenhang wird eine anthropologische Annahme wichtig. Aristoteles bindet nämlich den Sinn für das „Wahre und Gerechte" an die Natur, wenn er sagt, sie, das Wahre und Gerechte, seien von Natur aus stärker als das Gegenteil, womit er die dem Menschen eigentümliche Natur in ihrem Zusammenhang von Körper und Geist, physis S. 333.

${ }^{10}$ Protagoras, in Wilhelm Alfred CAPELLE, Die Vorsokratiker, Stuttgart, Kröner, 1963,

${ }^{11}$ Ibid., S. 327.

${ }^{12}$ ARISTOTELES, Topik, Hamburg, Meiner, 1992, S. 1 (100b). 
und logos, meint. ${ }^{13}$ Auch an dieser Stelle nimmt Aristoteles Gedanken der vorplatonischen und sophistischen Anthropologie auf, in der Bildung und Erziehung als eine in der Linie der Naturanlage liegende Vervollkommnung, nicht etwa Entfremdung gefasst wurden. Ich zitiere als ein Beispiel ein Diktum Demokrits: „Natur und Erziehung sind etwas Ähnliches. Denn die Erziehung formt zuvor den Menschen um, aber durch diese Umformung schafft sie Natur." "14 So erhalten wir den Grund für die Überzeugungskraft alles Natürlichen, dafür also, dass die Natur zum Beweistopos für alle möglichen Zweifelsfragen bis in politische Werbung und Propaganda unserer Gegenwart werden konnte, und ihr Gegenteil, die Unnatur, das Gemachte, Gefertigte, Technische sich mit geringerer Überzeugungskraft begnügen musste. Dass es in der Rhetorik dann gerade das pragmatische Herstellen im Vollzug der Regeln, also die ars ist, die dem Adressaten eine Sache zur natürlichen macht, kommt in dem paradoxen Grundsatz zum Ausdruck, den ich als Titel gewählt habe: ars est artem celare. Er ist viel älter als seine lateinische Formulierung. So versteht es sich für Aristoteles, dass die Téchné, also die Kunstfertigkeit nach der Natur sich richtet und zusammen mit entsprechender Bildung (paideía) „nur die Lücken ausfüllen soll, welche die Natur noch gelassen hat." ${ }^{\text {"15 }}$ Wir dürfen uns das ruhig wie bei einem Bilde vorstellen, in dem die Kunst das, was noch fehlt, ganz natürlich einträgt. Oder auf die Rede bezogen: „Daher ist es erforderlich, die Kunstfertigkeit anzuwenden, ohne daß man es merkt und die Rede nicht als verfestigt, sondern als natürlich erscheinen zu lassen - dies nämlich macht sie glaubwürdig [...].“16 Und dann folgt an dieser Stelle noch eine Ergänzung, die wir für meine weitere Gedankenführung jetzt schon im Kopfe behalten sollten. Wenn die Technik der Herstellung (hier dürfen wir den Ausdruck „Technik“ verwenden, weil er in diesem Kontext schon die Bedeutung annimmt, die uns heute geläufig ist) wenn also die Technik sichtbar, vielleicht dominierend erscheint, dann macht das die Rede unglaubwürdig, „denn die Zuhörer nehmen wie gegen jemanden, der etwas im Schilde führt, Anstoß daran $[\ldots]$...17

Müssen wir diesen Schein der Natürlichkeit, der durch das Verbergen der Kunst zustande kommt, jetzt aber nicht als eine Lüge bezeichnen, da ja täuschende Absicht dahinter steht und der Redner sich dessen sehr wohl bewusst ist? Ich komme jetzt zu einem weiteren und zwar höchst wichtigen Diskussionspunkt. Dem Redner geht es nicht um wahre Erkenntnis, sondern um glaubwürdige, überzeugende Wirkung. Indem er (um es an den partes orationes zu illustrieren) dem natürlichen Redeaufbau folgt und z.B. die Einleitung nicht mitten in die Argumentation oder die peroratio, die

\footnotetext{
${ }^{13}$ ARISTOTELES, Rhetorik, S. 10 (1355a).

${ }^{14}$ Ibid., S. 11 (1355b).

15 Demokritos, in Hermann Diels, Walther KRANZ (Hrsg.), Die Fragmente der Vorsokratiker, Berlin, Weidmann, 1951, Bd. 2, S. 68 (B 33).

${ }^{16}$ ARISTOTELES, Politik, Reinbek, Rowohlt, 1994, S. 339 (1337a).

${ }^{17}$ ARISTOTELES, Rhetorik, S. 170.
} 
Schlussrede, an den Anfang stellt, auch in seinem Redefortgang keine Stockungen oder Umkehrungen zulässt, weil die Natur keine Sprünge kennt, bewirkt er eine Glaubhaftigkeit, die für den Adressaten überzeugungskräftiger ist als das Abweichen vom natürlichen Gang der Rede. Die bleibt aber, wie sehr auch immer sie sich an der Natur orientieren mag, Sprachwerk; niemals kann ihr gelingen, was man an Zeuxis rühmte, der auf einem Bilde die Weintrauben so natürlich gemalt hatte, dass lebendige Tauben danach pickten. (Was Hegel zu der treffenden Bemerkung veranlasste: ,anstatt Kunstwerke zu loben, weil sie sogar Tauben [...] getäuscht, gerade nur die zu tadeln sind, welche $[\ldots]$ nur eine so niedrige Wirkung [...] als das Letzte und Höchste zu prädizieren wissen." $)^{18}$

Ich wiederhole es: indem die Rede Sprachwerk ist, kann ihre Wirkung auch nicht über die der Sprache mögliche hinausgehen. Adornos philosophiekritische Bemerkung zielte schon in diese Richtung. Deren Quintessenz war schon sehr früh rhetorischer Gemeinbesitz. Eines der ersten Zeugnisse stammt von dem völlig zu Unrecht von Platon verlästerten Gorgias aus Leontinoi. „Denn was man sah, wie sollte man dies durch Rede aussprechen? [...] Und es spricht, wer spricht - aber nicht eine Farbe und auch kein Ding.“19 Diese Stelle aus dem Fragment über das „Nichtseiende“ ist auch deshalb interessant, weil sie wohl die Sprachtheorie des frühen Nietzsche mit inspiriert, vielleicht angestoßen hat. Bei Worten komme es „nie auf die Wahrheit an“ ${ }^{\text {20 }}$, ist ja seine These. Dann folgt die Begründung: „Ein Nervenreiz wird zuerst übertragen in ein Bild! Erste Metapher. Das Bild wieder nachgeformt in einen Laut! Zweite Metapher.“21 In Metaphern formt Sprache aber nichts anderes als Eindrücke nach. „Nicht die Dinge treten ins Bewusstsein", so lautet an anderer Stelle das konzise Resümee ganz nach Gorgias“ Vorgabe, „sondern die Art, wie wir zu ihnen stehen“'22. Oder mit anderen Worten: unsere Meinung über sie. ${ }^{23}$

Womit ich nach einem kleinen Umweg wieder bei der Hauptsache gelandet bin. Denn die Rhetorik hat es mit Meinungen zu tun, nicht mit Wissen, das ist schon in der Definition enthalten, die ich anfangs ausführlich zitierte, und in der Aristoteles hervorhob, dass nämlich von rhetorischem Belang „nur solche Dinge [sind], welche sich allem Anschein nach auf

${ }^{18}$ Ibid.

${ }^{19}$ Georg Wilhelm Friedrich Hegel, Ästhetik, Hg. v. F. BASSENGE, 2 Bde., Frankfurt/ M., o.J., Bd. 1, S. 52. S. 51

${ }^{20}$ Gorgias von LeOnTINOI, Reden, Fragmente und Testimonien, Hamburg, Meiner, 1989,

Friedrich NIETZSCHE, Ueber Wahrheit und Luege im außermoralischen Sinne, in Friedrich NiETZSCHE, Kritische Studienausgaben in 15 Bänden, München, Dtv, 1999, Bd. 1, S. 879 .

${ }^{22}$ Ibid., S. 879.

${ }^{23}$ Friedrich NiETZSCHE, Werke. Großoktavausgabe in 19 Bdn, Leipzig, 1894ff, Bd. 18, S. 249. Einen sehr guten Überblick über Nietzsches Gedankenentwicklung gibt: Josef KOPPERSCHMIDT, "Nietzsches Entdeckung der Rhetorik", in Josef KOPPERSCHMIDT, Helmut SCHANZE, Nietzsche oder ,Die Sprache ist Rhetorik“, München, Fink, 1994, S. 39ff. 
zweierlei Weise verhalten können“،24. Ohne dass er es nach Philosophenweise ausdrücklich hervorhebt, vernehmen wir im Hintergrund Protagoras, der das nicht anders gesehen und kaum anders formuliert hatte, dass sich nämlich die Rhetorik mit Themen beschäftige, über die man mit gleichem Rechte nach beiden Seiten (also in einander entgegengesetztem Sinne) disputieren könne. $^{25}$ Das hier jeweils zugrunde gelegte Modell scheint mir das der Gerichtsrede zu sein, die als Gattung auch historisch am Anfang der Redekunst steht. Die Vielfalt gleichberechtigt nebeneinander bestehender Meinungen ist ihr Lebenselement, und es ist von entscheidender Bedeutung, dass jede nur eine Vermutung vorschlägt oder eine Widerlegung hervorbringt, die bessere oder treffendere Alternative sich aber nicht von selber zeigt. Im Gerichtsverfahren schlägt sich musterhaft und übersichtlich nieder, was den Normalfall unseres Lebensvollzugs in allen öffentlichen und privaten Angelegenheiten ausmacht. Auch die Wissenschaften sind da nicht ausgenommen. Der von Thomas S. Kuhn in seiner Untersuchung wissenschaftlicher Revolutionen so genannte „Paradigmenwechsel“ verweist auf die bloße Wahrheitsähnlichkeit und Zustimmungsabhängigkeit sogar naturwissenschaftlicher Theorien. Es liegt in dem germanistischen Kontext, in dem wir uns in diesem Colloquium bewegen, nahe, auf die Auseinandersetzung zwischen Goethe und Newton zu erinnern oder daran, dass sowohl die Korpuskel - wie die Wellentheorie des Lichts jeweils plausible Erklärungen liefern. Auch für Meinungsdivergenzen dieser Art hatte übrigens Protagoras Aufschluss gegeben, der ganz modern anmutet. Im Stoff der Materie selber liege der Grund, sie sei (und hier ist Heraklit nicht weit) ständig „im Fluß begriffen“, so dass verschiedene Zustände dicht nebeneinander existieren. „Die Menschen“, so vermutete er, „erfaßten bald das eine, bald das andere [davon], entsprechend ihren eigenen verschiedenen Zuständen.'“26

Das ist ersichtlich ein unsicherer Boden, dessen weiterer Erkundung sich ein ganzer Seitenzweig der Rhetorik (die Wissenschaftsrhetorik) widmet. Ich möchte unsere Aufmerksamkeit aber auf die eigentümlichen Merkmale der Meinung zurücklenken, die der Lüge Vorschub leisten. In einer Sprichwortsammlung von 1810 steht das bezeichnende Exempel: „Der Meyner und der Lügner sind zwey Brüder.“27 Die Meinung, doxa nannten sie die Griechen, kann wie wir ja wissen, nur zu wahrscheinlichen, wahr scheinenden, Schlüssen kommen, deswegen sind gleichberechtigte Meinungen möglich, die ein evidentes Urteil ausschließen würde. Das scheint eine negative Bestimmung und führt uns doch auf eine positive Spur: die Meinung ist offen, sozusagen durchlässig, sie zieht Gegenmeinungen heran, provoziert sie auch, muss auf jeden Fall immer mit ihnen rechnen, weil ihr die

\footnotetext{
${ }^{24}$ ARISTOTELES, Rhetorik, S. 16 (1357a).

${ }^{25}$ Protagoras, in CAPELLE, Die Vorsokratiker, S. 326.

${ }^{26}$ Ibid., S. 330f.

${ }^{27}$ Johann Michael SAILER, Die Weisheit auf der Gasse. Deutsche Sprichwörter, 1810, ND, Nördlingen, Greno, 1987.
} 
eigene Relativität innewohnt. Unser Sprachgebrauch ist in diesem Falle ein guter Wegweiser. Wir reden davon, dass sich die öffentliche Meinung im Fluss befinde, dass wir unter den gegebenen Verhältnissen diese oder jene Meinung favorisieren oder dass jeder halt seine eigene Meinung habe. „Alles Meinen“, so fasst Ernst Bloch ihr wichtigstes Merkmal in einem Satz zusammen, alles Meinen „schießt über das Feste hinaus, worin es sich gerade befindet.“28 Diese Deutung schlägt vor, das Meinen als produktiven Denkvorgang zu betrachten, prozesshaft auf Verbesserung, Bereicherung, Vermehrung durch andere, gerade gegenteilige Meinungen angelegt.

Ich glaube, dass die klassischen Autoren nichts anderes im Sinne hatten, wenn sie von Meinung sprachen. Als ein Beispiel zitiere ich Cicero, und zwar aus dem 2. Buch von De oratore, in dem immer wieder aus der Gerichtsrede heraus allgemeine Folgerungen gezogen werden. „Die ganze Tätigkeit des Redners“, lässt der Autor Antonius ausführen, „gründet sich auf Meinungen (opiniones) und nicht auf Wissen (scientia). Denn einmal reden wir

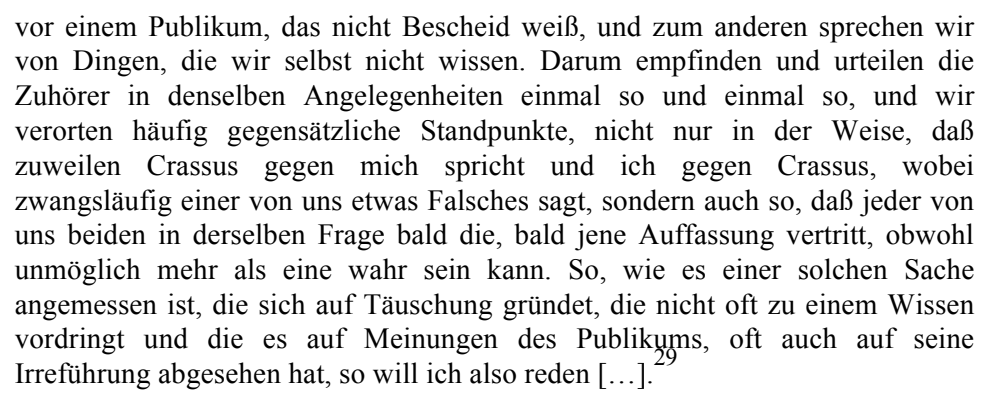

Was ich da von Cicero so ausführlich zitiert habe, könnte nun jede Denunziation aus platonischem Geiste bestätigen, und Cicero scheint es geradezu darauf angelegt zu haben. Liest man aber genau, so fasst er auch an dieser Stelle das Meinen als ein dialektisches Fortschreiten durch Widersprüche auf. Dazu ist freilich eine Voraussetzung nötig, über die schon in der Antike viel diskutiert wurde. Meinung und Gegenmeinung (um den Pluralismus der Meinungen modellhaft zu vereinfachen) müssen sich nämlich in einem Verhältnis zueinander befinden, der das Konkurrieren überhaupt fruchtbar macht und einen Fortschritt in der Lösung eines, sagen wir juristischen oder politischen Problems bringt. Sie müssen sich, um es mit einer uns geläufigen Metapher zu sagen, auf Augenhöhe begegnen, und das ist oftmals nicht von vornherein gegeben. So befindet sich nicht nur der Redner im Nachteil, der eine sehr viel schwächere Meinung vertritt als sein Gegner, weil er z.B. die herrschende Gesetzesauslegung gegen sich hat oder der im Publikum herrschenden Meinung entgegen treten muss. Auch die Problemlösung selber gerät in Gefahr, nicht zum optimalen Ergebnis zu

\footnotetext{
${ }^{28}$ Ernst BLOCH, Logos der Materie. Eine Logik im Werden, Frankfurt/ M., Suhrkamp, 2000, S. 387.

${ }^{29}$ CiCERO, De oratore. Über den Redner, Stuttgart, Reclam, 1976, S. 225 (II, 30).
} 
finden. Protagoras, der darüber nachgedacht hatte, verlangte daher vom Redner eine eigene Kunstfertigkeit, nämlich „die schwächere Sache zur stärkeren “30 machen zu können. Damit war eine Debatte entfacht, über deren Heftigkeit sogar ihr an Widerworte gewohnter Urheber wohl selber verwundert war. Denn hieraus resultiere doch, so lautete ein gewichtiges Widerwort, „daß die Menschen mit Recht das Anerbieten des Protagoras unwillig zurückweisen; denn es ist Lüge [...]. ${ }^{\text {31 }}$ Die Invektive stammt ausgerechnet von Aristoteles, der schon ganz zu Beginn seines „RhetorikBuches“ doch so unmissverständlich fordert: „Weiterhin muß man in der Lage sein, eine gegenteilige Ansicht (doxa) überzeugend darzulegen [...]. ${ }^{\text {،32 }}$ Bleibt zu fragen, wie das gelingen soll, wenn nicht derjenige, der die schlechtesten Karten hat, sie so einsetzt, dass sie dennoch das gegnerische Blatt zu übertrumpfen vermögen?

Sehen wir uns aber die Verhältnisse an drei Beispielen an. Die beiden ersten entnehme ich Manfred Fuhrmanns Analyse von Ciceros prozesstaktischem Geschick. ${ }^{33}$ Die früheste Rede Ciceros, die uns überliefert ist, behandelt einen zivilrechtlichen Streitfall, und zwar das „Plädoyer ,Pro Quinctio“". Es geht um Erbschaftsangelegenheiten ziemlich komplizierter Natur und Cicero benutzt deren Schwierigkeit, indem er vorgibt sie sachlich klären zu wollen, zu anderen Zwecken; mit Fuhrmanns Worten:

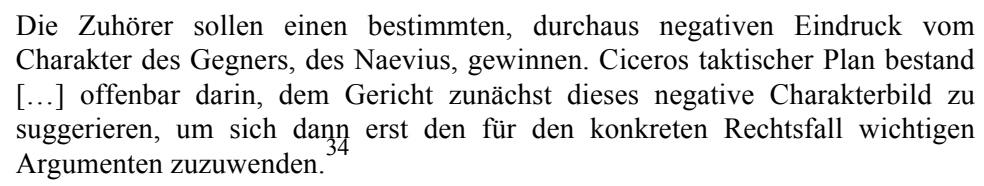

Wir dürfen das durchaus als bewusstes Täuschungsmanöver bewerten, ebenso wie das zweite Beispiel, das ein noch extremeres Vorgehen zeigt. Es ist eine der berühmtesten Reden Ciceros: „Pro Milone“ gehalten, d.h. für einen Mann, den zu verteidigen den schwierigsten Vertretbarkeitsgrad bedeutete. Die äußeren Umstände waren höchst prekär, die Republik rückte ihrem Untergang immer näher, Bandenkämpfe waren im Jahr 52 längst an der Tagesordnung in Rom. Milo und Clodius waren konkurrierende Bandenführer, die sich auf offener Straße befehdeten, bei einem ihrer Gefechte blieb Clodius auf der Strecke und Milo wurde des Mordes an ihm angeklagt. Erschwerend kam für Cicero hinzu, dass Clodius, wie jedermann wusste, auch sein Erzfeind gewesen war. Hören wir, wie Fuhrmann Ciceros Vorgehen beschreibt:

\footnotetext{
${ }^{30}$ Protagoras, in CAPELLE, Die Vorsokratiker, S. 325.

${ }^{31}$ ARISTOTELES, Rhetorik, S. 161 (1402a).

${ }^{32}$ Ibid., S. 10 (1355a).

${ }^{33}$ Manfred FuHRmAnN, Redekunst am Beispiel Ciceros, Stuttgart/ Düsseldorf/ Leipzig, Klett, 1997.

${ }^{34}$ Ibid., S. 57.
} 
Der Vorwurf (Clodius erschlagen zu haben) bestand zu Recht, und der Einwand des Verteidigers, Milo habe in Notwehr gehandelt, ließ sich, wie zuverlässig überliefert ist, mit dem tatsächlichen Ablauf des Geschehens nicht in Einklang bringen. Die taktischen Kunstgriffe, mit denen Cicero der heillosen Lage zu begegnen suchte, laufen auf zweierlei heraus. Er gab ein entstelltes Bild vom Sachverhalt und war umso mehr darauf bedacht, für Stimmigkeit innerhalb der Rede, zwischen Sachverhaltsschilderung und Beweisführung, zu sorgen; er suchte die Schwächen der rechtlichen Argumentation durch eine politische Deutung des Falles zu überspielen, die er als extra causam befindlich (etwa: über den rechtlichen Aspekt der Sache hinausführend) ausgab [...].

Das dritte Beispiel dürfte vielen von uns vertrauter sein, so dass ich nur darauf anzuspielen brauche. Es ist die 4. Catilinarische Rede, eine politische Rede also, die Cicero vor dem Senat am 5. Dezember 63 gehalten hat. Sie galt der Frage, was nun mit den in Rom verhafteten Verschwörern zu geschehen habe, zwei Anträge lagen vor: Caesar plädierte für Verbannung, Iunius Silanus, der schon gewählte nächste Konsul, für die Todesstrafe. Der noch amtierende Konsul Cicero legt vor den „versammelten Vätern“ (den Senatoren) die Anträge scheinbar unparteiisch dar, malt dann aber wie in einer Vision die von den Vaterlandsverrätern beabsichtigte Zerstörung des Staats und den Untergang Roms höchst drastisch aus mit den Mitteln des ad oculos ponere, des Vor-Augen-Stellens: die brennende Stadt, die gemetzelten Bürger, die geschändeten Frauen, Kinder, Priesterinnen. ${ }^{36}$ Die große Mehrheit der Senatoren entschied sich für die Todesstrafe.

Das sind auf Anhieb höchst irritierende Beispiele und man könnte sie leicht vermehren. Sie haben wesentlich zu dem negativen Charakterbild beigetragen, das Cicero zumal in der deutschen Geschichtsschreibung des 19. Jahrhunderts verpasst wurde. Eines Jahrhunderts freilich, das von der Rhetorik kaum noch mehr als die Figurenlehre und vielleicht den Redeaufbau (dispositio) wusste. Die dialektischen Anteile (Topik) hatte schon in der Renaissance die Philosophie sich angeeignet, das geschieht auch im 19. Jahrhundert, ist aber bis heute noch nicht ausreichend gewürdigt worden. Ciceros rhetorisches Vorgehen ist ja einer doppelten Auslegung fähig. Moralisch zunächst, im Sinne des ersten und wohl wirkungsmächtigsten Lügentheoretikers Augustinus, der bekanntlich vor seiner Bekehrung ein berühmter Rhetor gewesen war und sich als Redelehrer betätigt hatte. Lüge hatte er definiert als „eine bewußt unwahre, mit der Absicht zu täuschen gemachte Aussage".37

Eine zweite Auffassung von Ciceros persuasiven Verfahren geht von der rhetorischen Wirkung aus, die - besonders extrem im Streitfalle - die widersprechende Meinung im Interesse eines optimalen Ergebnisses zur Geltung bringt. Das persuasive Prinzip hatte Protagoras, hatte auch Aristotles beschrieben. Im 19. Jahrhundert - so meine These - bemächtigt sich seiner

\footnotetext{
${ }^{35}$ Ibid., S. 69.

${ }^{36}$ Vgl. CiCero, Die Politischen Reden, München, Artemis, 1993, Bd. 1, S. $477 \mathrm{ff}$.

${ }^{37}$ Augustinus, Die Lüge und gegen die Lüge, Würzburg, Augustinus Verlag, 1953, S. 7.
} 
auf eigenartige Weise Hegels Philosophie, des näheren seine Dialektik. Ich denke, dass ich ihr folgend, die rhetorische Situation genauer als bisher möglich beschreiben kann. Auch ist sie ja dadurch charakterisiert, dass keine Bestimmung schon endgültig genannt werden kann, und das unmittelbar jeweils Angenommene, so Hegel in seiner Würdigung des antiken Skeptizismus, „nichts Festes, nichts an und für sich ist.“38 Da „Meinung“, enthält dieses zunächst bloß Angenommene die eigene Vorläufigkeit und das anders Meinende, gegenteilig Meinende schon in sich. Zugespitzt formuliert, es ist Spruch und Widerspruch in einem, jedoch nicht um des artistischen Spieles, sondern um der bestmöglichen Lösung eines Problems willen, ob es sich nun um ein juristisches oder politisches Problem handelt. In einem seiner schönen Gleichnisse hat Hegel den Widerspruch auch als Verstellung des Geistes, seines versteckten Wühlens sogar geschildert. „Bisweilen erscheint dieser Geist nicht offenbar, sondern treibt sich, wie die Franzosen sagen, sous terre herum. Hamlet sagt vom Geiste, der ihn bald hier-, bald dorthin ruft: du bist mir ein wackerer Maulwurf, denn der Geist gräbt oft wie ein Maulwurf unter der Erde fort und vollendet sein Werk. Wo aber das Prinzip der Freiheit sich erhebt, da tritt Unruhe, ein Treiben nach außen, ein Erschaffen des Gegenstandes ein, an dem der Geist sich zu zerarbeiten hat. ${ }^{\text {(39 }}$ Hegels Diktum von der „List der Vernunft ${ }^{\star 40}$ hat es sogar zum geflügelten Wort gebracht.

Ich will mich nun nicht in eine Diskussion der Hegelschen Geschichtsdialektik vertiefen und verlieren und nur soviel festhalten, dass mit Hegel der Geist des Widerspruchs auf eine Art gedacht wird, wie ihn sich die Rhetorik seit Protagoras dienstbar gemacht hat. Und dies durchaus schon in einem bewusst außermoralischen Verständnis, das aber nicht etwa auf eine Herrschaft der Lüge abzielt, sondern deren unterirdisch wirkende Kraft ausnützt. Gleich jenem Gesellen, „der (wie es im Prolog im Himmel des Faust-Dramas heißt als habe Hegel es diktiert) „,der reizt und wirkt und muß als Teufel schaffen. ““1 Derart erscheint auch die Lüge eingesetzt, um für offene Fragen oder strittige Probleme ein bestmögliches Ergebnis zu erzielen. Sie wird zum Mitproduzenten der Wahrscheinlichkeit und arbeitet dann an ihrer eigenen Aufhebung. Denn sie hilft, das, was dunkel und verworren ist, zur Klarheit zu führen, um es noch einmal in der Sprache des Goethe-Stücks zu sagen. ${ }^{42}$ Es gibt auch bei einem anderem uns zeitlich (und hier in Aix auch geographisch) näher liegenden Autor eine Stelle, die wie eine Erläuterung unseres Themas nach rhetorischem Gesichtspunkt wirkt. Ich meine Camus' La Chute (Der Fall), und zwar des näheren den Beginn des letzten Kapitels, in dem der „Buß-Richter“ Clamence seinem Zufallsbekannten aus dem „Mexico-City“ erzählt, wie er zum Papst gewählt wurde.

\footnotetext{
${ }^{38}$ Georg Wilhelm Friedrich HegEL, Vorlesungen über die Geschichte der Philosophie, in ders., Werke in 20 Bdn, Frankfurt/M., Suhrkamp, 1969, Bd. 2, S. 395

${ }^{39}$ Georg Wilhelm Friedrich HeGEL, Werke, Gesamtausgabe 1832-1845, Bd. 9, S. 73.

${ }^{40}$ HeGel, Werke in 20 Bdn, Bd. 6., S. 452.

${ }^{41}$ Johann Wolfgang GOETHE, Sämtliche Werke, Zürich, Artemis, 1977, Bd. 5, S. 152.

${ }^{42}$ Ibid., S. 151, so Hegel.
} 


\begin{abstract}
Sehen Sie, ein Bekannter von mir pflegte die Menschen in drei Gruppen einzuteilen: die einen möchten lieber nichts zu verbergen haben als lügen müssen; die anderen möchten lieber lügen als nichts zu verbergen haben; und die Dritten schließlich lieben das Lügen und das Verbergen gleichermaßen. Ich überlasse es Ihnen, die Kategorie zu wählen, in die ich am besten passe. Was tut's übrigens? Bringen die Lügen einen nicht letzten Endes auf die Spur der Wahrheit? Und zielen meine Geschichten, die wahren so gut wie die unwahren, nicht alle auf den gleichen Effekt ab, haben sie nicht alle den gleichen Sinn? Was hat es da zu besagen, ob ich sie erlebt oder erfunden habe, wenn sie doch in beiden Fällen für das bezeichnend sind, was ich war und was ich bin? Man durchschaut den Lügner manchmal besser als einen, der die Wahrheit spricht. Die Wahrheit blendet wie grelles Licht: Wohingegen die Lüge ein milder Dämmerschein ist, der jedem Ding Relief verleiht. Nun, ob Sie es glauben oder nicht, ich wurde in einem Gefangenenlager zum Papst gewählt.
\end{abstract}

Wenn Camus seinen Protagonisten an dieser Stelle die Lüge als eine Art Ferment der Wahrheit begreifen lässt, so befindet er sich offensichtlich im Einklang mit einer bis in ihre Anfänge zurückreichenden rhetorischen Tradition, die gelegentlich verschüttet, von der Philosophie denunziert, aber nie ganz unwirksam geblieben ist. Als rhetorische Kunst betrachtet und im artem-celare-Prinzip aufgehoben, vermag sie zwar im strengen und logischen Sinne nicht Wahrheit zu produzieren, denn die ist, mit Lessings Wort, nur bei Gott allein $^{44}$, auf jeden Fall allem menschlichen Wissen unzugänglich. Doch entfaltet sie, wie die Cicero-Beispiele zeigen sollen, ihre strategische Wirksamkeit im Prozess der Meinungsbildung. Insofern auch ihr, wie absolut sie immer auftreten mag, allein der Status einer Meinung zukommt, provoziert sie notwendig Gegenmeinungen und das umso energischer, je deutlicher ihre eigentliche Absicht der Täuschung sukzessive hervortritt. Da zuletzt alle rhetorische Anstrengung dem Ziel des gelingenden Lebens in politischer Gemeinschaft (der Polis nach aristotelischem Verständnis) dient und nur unter dieser Voraussetzung auch berechtigterweise aus der Zustimmung der Adressaten den Prüfstein ihrer Sätze und Urteile beziehen kann, hat die allein dem individuellen Vorteil dienende Lüge in der Tat nur kurze Beine und scheitert, wie vollkommen die egoistische Absicht auch immer kaschiert wurde.

Doch muss man hier wohl noch kritisch fragen, ob dieses Resümee nicht im schlechten Sinne idealistisch, also abstrakt, mindestens realitätsfern und somit uneinlösbar bleibt. Tatsächlich gewinnt es praktische Plausibilität erst im Kontext mit einem Rhetorik-Begriff, der nicht zu jeder Zeit und unter allen Bedingungen auch gegeben war und ist. Daher müssen wir uns daran erinnern, dass die Rhetorik ein Kind demokratischer Verhältnisse ist und auch Aristoteles sie so gesehen hat: im Kontext seiner Politik und ihrer Lehre von der besten Staatsverfassung (eine „,authentische bürgerliche Verfassung“, hat

\footnotetext{
${ }^{43}$ Albert CAMUS, Der Fall, Reinbek, Rowohlt, 2012, S. 99f.

${ }^{44}$ Gotthold Ephraim Lessing, Werke, München, Hanser, 1979, Bd. 8, S. 32f.
} 
sie Sternberger genannt ${ }^{45}$ ). Der alte Topos von der Zusammengehörigkeit von Rhetorik und Republik gewinnt in meiner Fragestellung neue und, denke ich, tiefere Bedeutung. Er verweist die Rhetorik auf die Praxis, in der RednerInteresse und Adressaten-Interesse übereinkommen: auf das Herausprozessieren der bestmöglichen Handlungsmaxime durch die argumentative Prüfung der Optionen von allen Seiten.

Woraus eine Rhetorik-Konzeption folgt, die trotz Protagoras und Aristoteles selten unangefochtene Geltung besaß und auch heute landläufigem Verständnis widerspricht: die Gründung der Rhetorik auf den Dialog, nicht auf den Monolog. Das ist viel mehr als eine bloß instrumentelle Unterscheidung. Schon in der Antike schälten sich beide Modelle erkennbar deutlich heraus, und wenn man, wie es bei Modellen nicht anders sein kann, auf vereinfachende Weise ihre geschichtliche Verortung bestimmen will, so favorisierte die griechische Rhetorik das dialogische, die römische Rhetorik das monologische Modell. In der einen Kultur ist der Redner Mitproduzent in einem umfassenden Meinungsbildungsprozess, im anderen fungiert er als Meinungs- und Handlungsführer. ${ }^{46}$ Die beiden Rhetorikkonzepte überschneiden sich an manchen (vor allem redepraktischen) Stellen, auch gibt es prominente (Cicero ist dafür ein Beispiel) Synthetisierungsversuche, ohne dass aber ihr fundamentaler Unterscheid eingeebnet werden könnte.

Er radikalisiert sich kritisch bis zum Antagonismus, konnte aber aus mehreren Gründen lange verdeckt bleiben. Dazu gehört auch die bis in die Französische Revolution und die Gründung der USA reichende Dominanz der römischen Rhetorik. Doch in der Adelsrepublik Roms finden wir eine andere gesellschaftliche und politische Struktur vor als in den griechischen Stadtstaaten. Hegel hat den „Räuberfang“ des römischen Staates, seine auf Überwältigung der Nachbarn, auf Gewalt und Krieg beruhende Stiftung dafür verantwortlich gemacht, dass „,nicht ein sittlicher, liberaler Zusammenhang [wie in Athen], sondern ein gezwungener Zustand der Subordination ${ }^{\text {“47 }}$ den Zusammenhalt der Menschen zu gewährleisten hatte.

Wie auch immer man $\mathrm{zu}$ solchen idealtypischen geschichtsphilosophischen Modellbildungen stehen mag, in unserem Fall schärfen sie den Blick für das Verhältnis des römischen Redners zu seinem Publikum. Wobei ich sogleich hinzufügen möchte, dass auch in der griechischen Polis verschiedene Konzepte der rhetorischen Lebensordnung miteinander konkurrierten. Sie reichten vom Anspruch des Redners, einer S. 156.

${ }^{45}$ Dolf Sternberger, Drei Wurzeln der Politik, Frankfurt/M., Springer, 1978, Bd. 1,

${ }^{46}$ Ich folge in diesem Zusammenhang meinen Ausführungen in Gert UEDING, "Das Konzept des Redners als Meinungsführer in der römischen Rhetorik", in Christina KUHN, Politische Kommunikation und öffentliche Meinung in der antiken Welt, Stuttgart, Steiner, 2012, S. 151 ff. Sowie: Gert Ueding, "Dialogrhetorik", in Gert Ueding/ Gregor KalivodA (Hrsg.), Wege moderner Rhetorikforschung. Klassische Fundamente und interdisziplinäre Entwicklung, (,Rhetorik-Forschungen 21“), Berlin/ Boston, de Gruyter, 2013, S. 701.

${ }^{47}$ Georg Wilhelm Friedrich HegEL, Vorlesungen über die Geschichte der Philosophie, in ders., Werke in 20 Bänden, Bd. 12, Frankfurt/M. 1985, S. 346. 
politischen Elite anzugehören und über eine Technik zu gebieten, die in jeder Lage und vor jedem Gremium Zustimmung erzwingen kann, bis hin zu der protagoreischen Auffassung, dass Handlungsorientierung erst aus dem Wettstreit gleichberechtigter Meinungen zu gewinnen sei.

Was diese gleichsam liberale Seite anbelangt, so blieb es Aristoteles vorbehalten, ein komplexes Rhetorikmodell nach dem Muster seiner Polis, „des menschlich vereinbarten Staates“, zu entwerfen. Der Bürger ist darin „immer ein potenzieller Staatsmann“ und umgekehrt der Staatsmann einer, der ,abwechselnd regiere und gehorche“. ${ }^{48}$ Ich will mir in Anbetracht des Themas, das ich hier zu behandeln habe, wohl eine nähere Erörterung der staatsphilosophischen Grundsätze des Aristoteles ersparen, doch wenn wir von ihnen aus seine berühmte Definition der Rede im 3. Kapitel des ersten Buches der Rhetorik lesen, können wir auch das Verhältnis der drei an einer Rede beteiligten Instanzen besser verstehen. Redner, Redegegenstand und Publikum verhalten sich demnach so, dass die Richtung der Rede vom Adressaten bestimmt wird, der mitdenkt und miturteilt, also sich nicht als Teil eines passiv empfangenden Auditoriums versteht. ${ }^{49}$ Offensichtlich verkörpert jeder Bürger die Rollen des Redners und des Zuhörers in seiner Person: der Redner ist Mitzuhörer und der Zuhörer Mitredner.

Die übliche Redehandlung vor Gericht in den staatlichen Gremien lief ganz entsprechend den aristotelischen Vorstellungen ab. Während seiner Rede bezog der Redner den dafür bestimmten Platz innerhalb seines Auditoriums und räumte ihn wieder für den nächsten Bürger, der sich zu Worte gemeldet hatte. Auch als wahrscheinlich der Pnyx genannte Hügel zum Ort der Volksversammlung wurde, in kleisthenischer Zeit, saßen die zuhörenden Bürger zuerst sogar etwas höher als der Standort des Redners, später befanden sie sich fast auf gleicher Höhe mit ihm.

Derartige Dispositionen von Redner und Publikum wirken wie eine Metapher auf das herrschende Rhetorik-Verständnis. So wird es uns kaum verwundern, wenn wir in Rom ein anderes Schema erblicken. Welcher Versammlungsort in Rom und anderen römischen Städten auch immer vorgesehen war, wie Forum oder Tempelplätze oder der Circus Flaminius, der Redner stand immer deutlich höher als die Versammlung, zu der er sprach - ob auf den Rostra oder den Tribünen der Tempel. Pragmatischer Grund (nämlich die bessere Akustik) und symbolische Zeichenbedeutung (nämlich der rhetorischen Einwegkommunikation) ergänzen einander. „Die Gleichheit, auf die die Athener stolz waren“, resümiert Francisco Pina Polo, dem ich hier ein Stück weit gefolgt bin, diese Gleichheit „,bezeichnet Cicero als iniqua, da sie nicht die dignitas berücksichtige, die die Bürger voneinander unterscheide und jedem einen anderen Platz innerhalb der Gemeinschaft zuweise. ${ }^{\text {‘50 }}$

\footnotetext{
48 Dolf SternBerger, Herrschaft und Vereinbarung. Schriften III, Frankfurt/M., Suhrkamp, 1980, S. 118.

${ }^{49}$ Vgl. UEDING, ,Dialogrhetorik“, S. 703.

${ }^{50}$ Francisca Pina POLO, Contra arma verbis. Der Redner vor dem Volk in der späteren römischen Republik, Stuttgart, Steiner, 1996, S. 25.
} 
Um schon etwas mehr von der Reichweite zu ermessen, die Ciceros Begriff der Rhetorik von dem griechischen Vorbild entfernt, braucht man nur in das einleitende Gespräch zu blicken oder besser zu hören, mit dem Cicero die Lehr-Dialoge Über den Redner eröffnet. „In meinen Augen“, so lässt er Crassus sprechen, dem er hauptsächlich die eigenen Ansichten in den Mund gelegt hat, ,in meinen Augen gibt es ja auch nichts Herrlicheres, als wenn man es vermag, die Menschen durch die Rede in seinen Bann zu schlagen, ihre Neigung zu gewinnen, sie zu verleiten, wozu man will, und abzubringen, wovon man will." ${ }^{\text {51 }} \mathrm{Da}$ scheinen wir beinahe bei dem Gegensatz zum aristotelischen Rhetor gelandet zu sein.

Kein Zweifel, dass Ciceros Redner-Ideal die Verhältnisse der Adelsrepublik und seine eigenen politischen Ambitionen durchaus widersprüchlich reflektiert. Sobald er freilich den Musterfall des Gerichtsverfahrens anvisiert, ändern sich gerade auch für ihn Aufgabe und Selbstverständnis des Redners und zwar im Sinne klassischer griechischer Auffassung. Juristische Rhetorik verzichtet auch für Cicero keineswegs auf den Überzeugungsprozess, in ihrem Rahmen skizziert er bereits die Umrisse eines - dialogischen - Verhandlungsmodells. Antonius formuliert das in De oratore mit dem Satz: „So übernehme ich [...] vollkommen unparteiisch drei Rollen in einer einzigen Person, die meine, die des Gegners und die des Richters.“ Der lateinische Text nennt die drei Rollen „tris personas “" ${ }^{\text {(52 }}$.

Offensichtlich also fasst der Advokat Cicero den Redner als ein Ensemble von Verhältnissen auf, die er in seiner Rede zum Sprechen bringt. Alles zusammen genommen oszilliert der rhetorische Begriff der Rede bei den klassischen Theoretikern zwischen Merkmalen, die ihn von der dialogischen Dialektik unterscheiden oder ihr naherücken, ihr zumindest analog sind. Dass in der Rhetorik-Geschichte bis heute das monologische Verständnis von Rede als Gegenstand der Theorie und Unterweisung vorherrschen sollte, hat seine Gründe auch in der politischen Geschichte Europas. Öffentliche Rede verwirklichte sich in der Predigt, im Herrscherlob oder in der Kriegsrede, drei Gattungen, die keinen beratenden, sondern apodiktischen, auch propagandistischen Charakter haben: in ihnen konnte jede Lüge unwidersprochen bleiben, sie produzierte nichts als sich selber. Das Gespräch blieb dem lehrhaften, akademischen Dialog vorbehalten, der zwar für die Tradierung des Konzepts und seiner Techniken sorgte, aber praktisch folgenlos bleiben musste.

Womit ich zum Abschluss noch auf ein historisch besonders radikales Exempel monologischer Rhetorik-Theorie und -Praxis hinweisen möchte, worin die Eliminierung der Alternativen die Lüge aus allen produktiven Bindungen entfesselte und das artem-celare-Prinzip in psychische Gewalt übersetzte. Ich meine die nationalsozialistische Rhetorik, lange vorbereitet durch Rhetoriker wie Carl Schmitt, Ewald Geissler oder Maximilian Weller,

\footnotetext{
${ }^{51}$ CiCERO, De oratore, 130.

${ }^{52}$ CiCERO, De oratore, 28.
} 
die die Rhetorik total auf das Orator-Prinzip gründeten. „Das Endziel aber, dem der Redner über alle Widerstände hinweg zudrängt, ist: dass die Hörer so werden, wie er sie haben will. So denken, so fühlen, so wollen, so handeln. “53

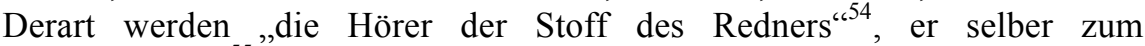
„Kampfredner“ ${ }^{\text {(55. }}$. Das alles sind Maximen Geisslers. Im Mittelpunkt der Theorie Wellers steht gleichfalls die Macht des Orators, die „seelenbezwingende, magische, dem künstlerischen, ja der Suggestion verwandte Wirkung ${ }^{656}$, die nichts anderes will als „eine Tat oder eine tatbereite Gesinnung hervorzurufen ${ }^{\text {‘57 }}$. Erfolgreichster Schüler solcher Lehren war Adolf Hitler, in Mein Kampf kann man ihre Spuren zum Teil wörtlich nachlesen. Ich zitiere ein Beispiel: „Die Macht aber, die die großen historischen Lawinen religiöser und politischer Art ins Rollen brachte, war seit urewig nur die Zauberkraft des gesprochenen Worts. ${ }^{\text {“58 }}$ Vom Eingehen auf den Zuhörer, der Vertrautheit mit ihm, spricht auch Hitler, doch ist kein Dialog damit gemeint. Die andere Meinung kennen bedeutet allein, den Schlachtplan eines Gegners kennen, den es zu schlagen gilt, mit Hitlers Worten: „Ich habe [...] gelernt, [...] dem Feinde die Waffe seiner Entgegnung gleich selber aus der Hand zu schlagen. ${ }^{\prime \prime 59}$ Die wenigen Belege mögen genügen, um die nationalsozialistische Rhetorik als den Exzess einer auf dem Orator-Prinzip fußenden Rede-Theorie zu decouvrieren.

Womit wir uns von einem Extrem rhetorischen Handelns wieder auf das Prinzip des „disputare in utramque partem“ verwiesen sehen, in dem (und darin liegt seine Stärke) noch die betrügerische Lüge dazu gebracht werden kann, als Kunstmittel zur Produktion ihres Gegenteils und damit zu ihrer Aufhebung zu wirken. Wie ein Sauerteig treibt sie hervor, was sie nicht wollte. Als Beispiel habe ich uns Cicero vorgestellt, ich hätte auch Sokrates, sogar den platonischen Sokrates nehmen können, der sich ständig einem Gesprächspartner in täuschender Absicht nähert, nämlich nichts oder weniger oder Falsches zu wissen und daher vorgeblich seiner Belehrung zu bedürfen. Man hat Sokrates mit seinen höheren pädagogischen Zwecken moralisch entlasten wollen. Dessen bedarf es nicht. Gibt es einen größeren Triumph über die Lüge als sie zum „Moment der treibenden Dissonanz“60 des Denkens, Redens, Handelns zu machen und derart gegen die Stilllegung menschlicher Tätigkeit einzuspannen? Die praktisch grundlegende

\footnotetext{
${ }^{53}$ UEDING, ,Dialogrhetorik“, S. 710.

${ }^{54}$ Ewald GEIßLER, Rhetorik. Zweiter Teil, „Deutsche Redekunst“, Leipzig, Teubner, 1918, S. $17 \mathrm{f}$. S. 53f.

Ewald GEIßLER, „Das Kampfgespräch“, in ders., Das gesprochene Wort, 3. Jg. 1940,

56 Maximilian Weller, Das Sprechlexikon. Lehrbuch der Sprechkunde und Sprecherziehung, Düsseldorf, Econ, 1957, S. 57.

${ }^{57}$ Maximilian Weller, Die freie Rede, Berlin, Der Deutschen Arbeitsfront, 1939, S. 94.

${ }_{58}^{58}$ Adolf HITLER, Mein Kampf, München, Zentralverlag der NSDAP, 1943, S. 116.

${ }^{59}$ Ibid., S. 522

${ }^{60}$ Ernst BLOCH, Subjekt-Objekt. Erläuterungen zu Hegel, Frankfurt/M., Suhrkamp, 1962, S. 93
} 
Voraussetzung dafür liegt freilich außerhalb rhetorischer Kompetenz: in der demokratischen Verfassung des Staates und den ihr entsprechenden Institutionen. 